\title{
Incorporación del tema de usabilidad en el diseño de sitios web en el curso de Multimedios
}

\section{Incorporation of usability in the web site design in the course Multimedios}

\author{
Iyubanit Rodríguez-Ramírez ${ }^{1}$ \\ Universidad de Costa Rica \\ Sede de Occidente \\ San Ramón, Costa Rica \\ Iyubanit.rodriguezramirez@ucr.ac.cr
}

\section{Recibido: 3 febrero 2013 Aceptado: 9 abril 2015 Corregido: 20 mayo 2015}

\begin{abstract}
Resumen: Este artículo tiene como propósito referirse a cómo se incorporó el tema de usabilidad en el curso de Multimedios de la Carrera de Informática Empresarial del Recinto de Grecia de la Sede de Occidente de la Universidad de Costa Rica. El estudio se llevó a cabo el I ciclo del 2011 y utilizó la estrategia didáctica llamada caso de estudio, en donde el estudiantado debía diseñar la interfaz de un carrito de compras por internet para una persona usuaria específica. Antes de hacer el diseño, se caracterizó a las personas usuarias finales y después se evaluó la usabilidad del diseño final. Los resultados de la evaluación del diseño de la interfaz por parte de las personas usuarias finales reflejó que los diseños mejoraron con respecto a la usabilidad de la aplicación, ya que el 81.25\% de estos recibió una nota de evaluación positiva por parte de las personas usuarias. Para determinar que los grupos de estudiantes asimilaron el tema de usabilidad se aplicó una encuesta basada en la técnica de la curva de aprendizaje, los resultados indicaron que el alumnado calificó con 9.07\% la pregunta sobre la experticia con el tema de usabilidad, después de haber realizado el caso de estudio.
\end{abstract}

Palabras claves: Usabilidad, estrategia didáctica, aplicaciones web, caso de estudio, curva de aprendizaje.

\begin{abstract}
The aim of this article is to expose how the topic of usability in the course Multimedia, which is part of the Business program imparted by Universidad de Costa Rica in Western Campus at Grecia location. The study took place on I semester 2011 and it used the teaching strategy called case study, where students had to design an Internet shopping cart graphical user interface for a specific user type. Before making the design, students characterized the selected users and then they had to assess the usability of the final design. The results of the evaluation of the design of the interface by the selected users reflected that people improved with respect to the usability of the application, since $81.25 \%$ of the designs received a positive assessment from users. To determine if students assimilated usability issues, a survey based on the technique of Learning Curve was applied. The results indicated that students scored $9.07 \%$ on the question of expertise with the topic of usability, after making the case study.
\end{abstract}

Keywords: Usability, teaching strategy, web applications, case study, learning curve.

1 Master en Computación e Informática de la Universidad de Costa Rica. Es docente e investigadora de la Carrera de Informática Empresarial de la Sede de Occidente de la UCR desde el año 2010. Ha participado como ponente en el congreso Latinoamericano de Informática (CLEI) en el 2012 y 2013 en el tema de género en computación e informática. También ha sido tutora de la representación del grupo de Olimpiadas de programación del concurso de la Association for Computing Machinery (ACM). Entre las publicaciones más recientes se encuentra: ¿Por qué ingresan tan pocas mujeres a la carrera de Informática Empresarial del Recinto de Tacares de la Universidad de Costa Rica. Un enfoque de género. Además, ha realizado investigaciones relacionadas con los temas de usabilidad en aplicaciones para personas adultas mayores y en modelaje y simulación del rendimiento de sistemas informáticos. Actualmente se encuentra realizando investigaciones en el área de los sistemas colaborativos acerca de cómo mejorar la comunicación entre abuelos y abuelas, y nietos y nietas que viven a distancia. 
Con la aparición del internet y actualmente con el surgimiento de las llamadas redes sociales como Facebook o Twitter, el perfil de las personas usuarias de las aplicaciones web está variando. La gama de perfiles van desde las personas usuarias expertas hasta aquellas que no tienen conocimiento en el uso de las computadoras, así como de personas que tienen dificultades para utilizar la computadora, ya sea por problemas físicos, mentales, tecnológicos o sociales. Por lo anterior la implementación y el diseño de la interfaz de un sitio web deben adecuarse a las características propias de las personas usuarias finales.

La usabilidad es definida por el estándar internacional ISO 9241-11 como "el grado en que un producto puede ser usado por determinados usuarios para conseguir objetivos específicos con eficacia, eficiencia y satisfacción en un contexto de uso especificado" (ISO, 1998, p. 2). Con la incorporación de la usabilidad a un diseño de la interfaz de un sitio web se pueden beneficiar tanto el personal de programación como las personas usuarias finales (Lorés y Granollers, 2004) de la siguiente forma:

- En el caso del personal de programación se reducen los costes de producción, mantenimiento y soporte (desarrollo) del sitio web. Al mismo tiempo se implementa un producto de mejor calidad que garantiza aplicaciones más competitivas, con menor necesidad de soporte y que pueden elevar las ventas del negocio.

- Con respecto a las personas usuarias, se da un sentimiento de confianza que produce la facilidad de uso del sitio, es decir, la persona visitante volverá en un futuro a utilizar el sistema y posiblemente recomendará el sitio a personas conocidas y amistades.

En este artículo se describe cómo se incorporó el tema de usabilidad en el curso optativo de Multimedios en el Recinto de Grecia que se encuentra dentro del programa de estudios de la carrera de Informática Empresarial y que se imparte en las sedes regionales de la Universidad de Costa Rica. Para la incorporación del tema de usabilidad se utilizó la estrategia didáctica llamada caso de estudio, el cual tiene como fin que el estudiantado pueda aplicar los conocimientos teóricos a un caso hipotético o real.

En este estudio se le proporcionó al alumnado un caso hipotético donde debe implementar y diseñar una interfaz para una aplicación de carrito de compras por internet para una persona usuaria específica. Después de implementar la aplicación, debe evaluar la facilidad de uso de la interfaz con la ayuda de una persona usuaria final. Para lo anterior se utilizó un método de evaluación llamado sistema de escala de usabilidad.

Con el fin de evaluar el aprendizaje por parte del estudiantado se aplicó una encuesta basada en la técnica llamada curva de aprendizaje, los resultados obtenidos se presentan y se analizan en esta investigación.

\section{Antecedentes}

En el año 1968 se fundó el Centro Universitario de San Ramón (Castro, 2009) y en 1977 se inaugura el Recinto Universitario de Grecia (Bolaños, 2013). Ambos recintos conforman la Sede de Occidente.

En la entrevista realizada a Sindy Porras, Directora del Departamento de Ciencias Naturales de la Sede de Occidente de la Universidad de Costa Rica (comunicación personal, 
2012), se menciona que la carrera Informática Empresarial con grado en bachillerato inició en 1998 en el Recinto de San Ramón, y empieza paulatinamente el cambio del grado de diplomado al grado de bachillerato en el Recinto de Grecia. En ese nuevo programa de estudios de grado bachiller es donde se incorpora el curso optativo Multimedios.

Multimedios pertenece al programa de la Carrera Informática Empresarial que se imparte en las seis sedes regionales de la Universidad de Costa Rica (UCR), es un curso optativo y tiene como objetivo principal que "el estudiante domine los conceptos, herramientas y metodologías necesarias para diseñar una aplicación web, así como ser capaz de desarrollar e implementar un sitio web" (Universidad de Costa Rica, 1998, p. 1).

\section{Descripción del problema}

El programa del curso de Multimedios no contaba con el tema de usabilidad en el diseño de aplicaciones web, lo anterior provocaba que el alumnado implementara diseños que resultaban, para la persona usuaria que los visitaba, una experiencia frustrante. Entre los problemas que se presentaban se citan:

a. La intencionalidad que el sitio deseaba trasmitir a la persona usuaria final era incorrecta o ambigua.

b. La navegación ofrecida por el sitio era inadecuada para el tipo de personas usuarias. Por ejemplo, a la población adulta mayor le puede resultar difícil utilizar el mouse o ratón, una solución es ofrecer otra alternativa de navegación como el teclado.

c. La cantidad de opciones presentadas en los menús del sitio web recargan la memoria de trabajo de algunas personas usuarias, lo aconsejable es utilizar entre 5 y 9 opciones dependiendo de las características de la persona usuaria final, pero en algunos casos el sitio web presentaba entre 20 a 30 opciones por pantalla.

d. Los elementos distractores de un sitio web (banners, anuncios, páginas de aterrizaje) no están equilibrados, lo cual podía provocar que la persona usuaria se distrajera de su objetivo a la hora de usar el sitio web.

e. Los diseños de la interfaz no contaban con los estándares dados por la World Wide Web Consortium (W3C).

Con la incorporación del tema de usabilidad dentro del temario del curso se buscaba que el alumnado realizara un estudio preliminar para caracterizar a la persona usuaria final, con el fin de conocer sus gustos, su conocimiento en el uso de las computadoras, sus limitaciones físicas, sociales y tecnológicas, entre otras. Por lo anterior se realizó un cambio dentro de los contenidos del curso y se incorporó el tema de usabilidad para el I ciclo de 2011.

\section{Descripción de la estrategia didáctica}

En esta se sección se presenta la descripción de la estrategia utilizada en el curso de Multimedios para incorporar el tema de usabilidad en aplicaciones web. 


\section{Estrategia didáctica: Caso de estudio}

La estrategia didáctica utilizada es la de casos de estudio. El Instituto Tecnológico y de Estudios Superiores de Monterrey indica que "un caso es la descripción de un hecho pasado que describe una situación compleja real" (Dirección de Investigación y Desarrollo Educativo del Sistema, 2000, p. 12). Esta estrategia tuvo sus inicios en 1914, cuando se utilizaba para la enseñanza de las leyes en el programa de Derecho en la Universidad de Harvard. Se pretendía que el alumnado buscara la solución a una historia concreta y la defendiera. Posteriormente, el método se extiende a otros campos como metodología docente (Dirección de Investigación y Desarrollo Educativo del Sistema, 2000; Martínez, 1999).

Se utiliza esta estrategia con el objetivo de que el estudiantado pueda aplicar los conocimientos teóricos a situaciones reales, lo cual permite un enlace entre la teoría y la práctica a la hora de realizar el caso de estudio. Lo anterior posibilita que el alumnado pueda construir su propio aprendizaje en un entorno similar al de la vida real (Dirección de Investigación y Desarrollo Educativo del Sistema, 2000; Servicio de Innovación Educativa, 2008).

La dinámica de este caso de estudio consiste en que el estudiantado debe analizar una situación problemática real o hipotética, con el fin de determinar las causas y los posibles efectos del problema y así poder plantear posibles soluciones al caso asignado. Al final se entrega un documento escrito de cómo realizó el análisis del caso de estudio, cómo lo implementó y las soluciones sugeridas al problema, para generar una discusión dentro del aula, donde el cuerpo docente guíe la actividad (Dirección de Investigación y Desarrollo Educativo del Sistema, 2000; Servicio de Innovación Educativa, 2008).

Entre las características de este método tenemos (Dirección de Investigación y Desarrollo Educativo del Sistema, 2000; Tobón, 2005):

1. Es interactivo y sumamente dinámico.

2. Está centrado fundamentalmente en el alumnado, el cual tiene un rol activo.

3. Ayuda al estudiantado a detectar problemas del mundo real y cómo afrontarlos.

4. Facilita el desarrollo de competencias argumentativas, comunicativas, propositivas e interpretativas.

5. Sirve como base de las discusiones en el aula en torno a las competencias que se esperan formar.

Es importante mencionar que se eligió esta estrategia didáctica de casos de estudio por ser un proceso metodológico flexible, el cual permite adecuarse a los objetivos del tema de usabilidad y condiciona el tipo de formación que se persigue enseñar al alumnado. Con la estrategia se pretende que el personal docente y el estudiantado participen en un clima positivo y en un ambiente colaborativo, donde puedan desarrollar los conceptos de manera grupal e individual (Martínez, 1999). Es importante mencionar que el tema de usabilidad involucra no solo aspectos tecnológicos sino también sociales y cómo las personas se relacionan con los sistemas que les ofrecen (Ramírez, 2012). Por lo tanto, el contacto con personas usuarias de la interfaz es necesario para que el alumnado participe de una experiencia real y comprenda los desafíos a los que pueda enfrentarse y así buscar soluciones adecuadas y correctas. 


\section{Papel de quienes se involucraron en el estudio de casos}

Cada uno de los individuos involucrados en el estudio de caso tiene un papel asignado. La descripción de cada uno de los papeles se presenta en esta sección.

Papel del personal docente. Ejerce varios roles en el desarrollo del caso de estudio:

1. Elaborar y proporcionar el caso de estudio a realizar.

2. Durante la realización del caso de estudio, es responsable de orientar o de guiar la actividad.

3. En la presentación del informe escrito, tiene como tarea mediar en la discusión de los resultados.

Papel del estudiantado. Desarrolla un papel activo e investigativo y debe realizar seis actividades fundamentales en el desarrollo del caso de estudio:

1. Leer y analizar el caso de estudio asignado.

2. Diseñar una interfaz de un sitio web para una persona usuaria específica y para una tarea concreta.

3. Evaluar la facilidad de uso del diseño de la interfaz con una persona usuaria final utilizando la técnica de evaluación sistema de escala de usabilidad (SUS, por su nombre en inglés), cuya explicación se presentará posteriormente.

4. Redactar un informe sobre los resultados obtenidos en la evaluación de la facilidad de uso de la interfaz y ofrecer posibles soluciones para mejorar su diseño.

5. Presentar el informe en clases y discutir sobre los resultados obtenidos del caso de estudio.

6. Finalmente, reflexionar sobre los resultados obtenidos y sobre la discusión que se llevó en clase.

Papel del contexto. A continuación se presenta los recursos necesarios para realizar el caso de estudio:

1. Una computadora para realizar el diseño de la interfaz de la aplicación web, con las herramientas necesarias para implementar este tipo de programa y para elaborar el informe escrito.

2. Material del tema de usabilidad dado por el personal docente en clases, así como la posibilidad de investigar el tema por cuenta propia, ya sea utilizando la biblioteca universitaria o el internet.

3. Cada grupo debe buscar una persona usuaria final que cumpla con las características que se especifican en el caso de estudio. Esta persona usuaria final participará en la evaluación de la usabilidad del diseño de la interfaz de la aplicación web. 


\section{Metodología}

En esta sección se va a exponer la metodología seguida para aplicar el caso de estudio del tema de usabilidad al curso de Multimedios.

\section{Grupo de estudio}

El grupo de estudio consiste en 33 estudiantes de cuarto año de la carrera Informática Empresarial del Recinto de Grecia, quienes matricularon el curso de Multimedios en el I ciclo del 2011. De esos 33 estudiantes 4 son mujeres (12.12\%) y 29 son hombres (87.87\%). Las edades del alumnado oscilan entre los 20 y 23 años.

Para efectos del caso de estudio se armaron 15 grupos de 2 personas y un grupo de 3 personas, en total quedaron 16 grupos de trabajo.

\section{Aplicación del caso de estudio}

La aplicación de este caso de estudio se realiza en cuatro etapas distintas: (1) entrega y explicación del caso de estudio, (2) creación del diseño de la interfaz, (3) aplicación de la técnica de evaluación de usabilidad y (4) presentación de los resultados a la clase del curso de Multimedios. En la figura 1 se presentan las etapas para la aplicación del caso de estudio.

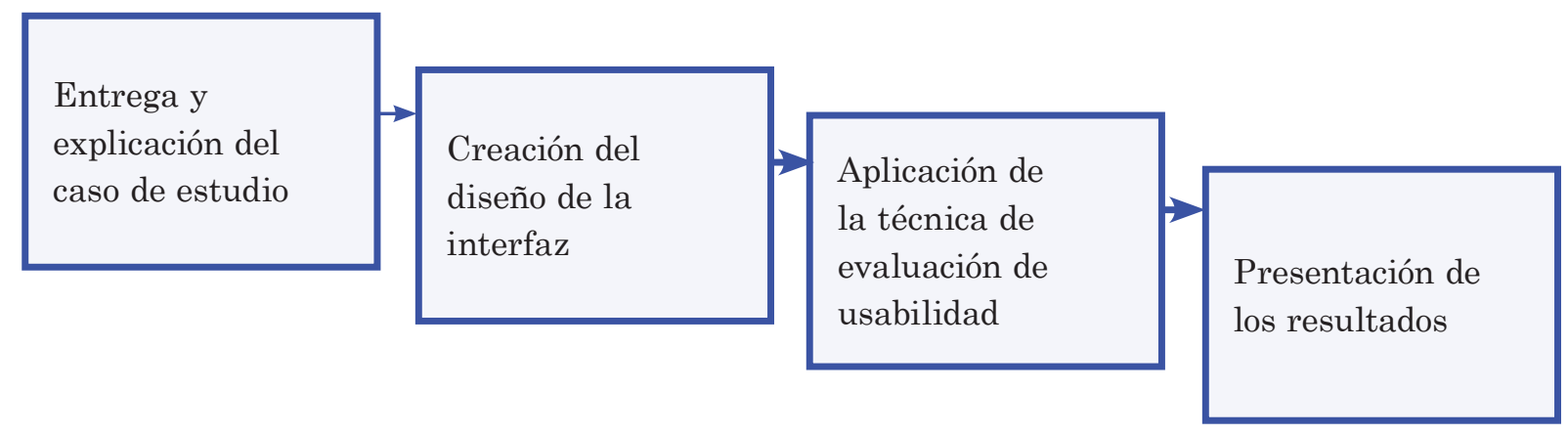

Figura 1. Etapas de la metodología seguida en el caso de estudio. Elaboración propia (2012).

Es importante mencionar que antes de realizar el caso de estudio, el personal docente impartió clases magistrales sobre los temas de usabilidad, de facilidad de uso, estándares de las W3C y de accesibilidad en aplicaciones web.

Etapa 1: Entrega y explicación del caso de estudio. El cuerpo docente entrega el enunciado del caso de estudio al alumnado y después se procede a leer, explicar y aclarar dudas. 
El enunciado del caso de estudio consiste en un caso hipotético donde el estudiantado debe realizar una aplicación web para una tarea específica. Una tarea es un objetivo que la persona usuaria final desea alcanzar al utilizar una aplicación. A todos los grupos se le asignó la misma tarea.

En el caso de estudio la tarea consiste en que la persona usuaria final pueda realizar compras de productos por medio de un carrito virtual. A cada grupo se le asignó una persona usuaria final específica, que podía ser: personas adultas mayores, infantes, personas empresarias, personas usuarias expertas en el uso de las computadoras y personas usuarias inexpertas en el uso de las computadoras, entre otras.

Etapa 2: Creación del diseño de la interfaz. El alumnado lee y analiza el caso, asumiendo la toma de decisiones debe identificar los puntos críticos en el planteamiento del caso: la tarea y la persona usuaria final que va a usar la aplicación.

Después se procede a diseñar e implementar una interfaz para una aplicación web. Para la creación de la interfaz deben utilizar los lenguajes XHTML y JavaScript, también hojas de estilo (CSS, por sus siglas en inglés) y material de usabilidad dado en clases anteriores.

En este punto se desea que el alumnado realice varias tareas:

1. Caracterizar a la persona usuaria final, analizar las particularidades propias tales como: las habilidades cognitivas (memoria), las habilidades de movilidad, el control de la motora fina, la experiencia en el uso de computadoras, la visión y la audición.

2. Buscar posibles alternativas para implementación de la interfaz de la aplicación web, basándose en los hechos del caso y en la caracterización de la persona usuaria.

3. Seleccionar en grupo la alternativa más apropiada para implementar la interfaz de la aplicación web, por medio de una discusión donde se intercambien conocimientos y experiencias de cada integrante.

4. Desarrollar la interfaz de la aplicación web siguiendo la alternativa seleccionada en el punto 3.

En la figura 2 se pueden apreciar los pasos seguidos en la etapa 2 de la creación de la interfaz de usuario de la aplicación web.

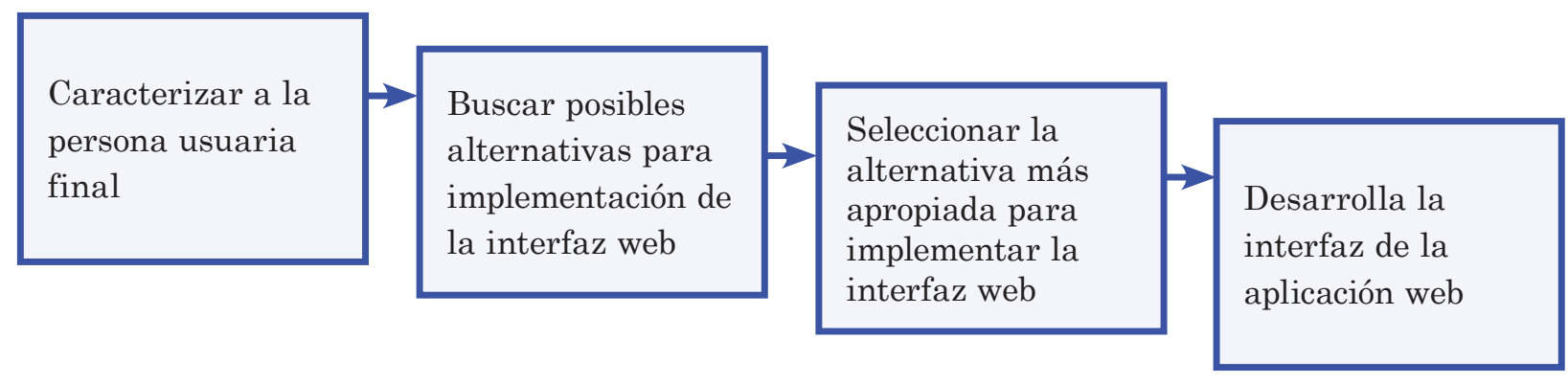

Figura 2. Pasos de la etapa 2: Creación del diseño de la interfaz. Elaboración propia (2012). 
Esta etapa es muy importante en el proceso del caso de estudio, debido a que la interfaz de una aplicación "es el principal punto de contacto entre el usuario y el ordenador; es la parte del sistema que el usuario ve, oye, toca y con la que se comunica. El usuario interacciona con el ordenador para poder realizar una tarea" (Lorés, Granollers y Lana, 2001, p. 11).

Las aplicaciones de software tienen la singularidad de no poder predecir de antemano si la facilidad de uso del diseño de una interfaz cumple con los requerimientos propios de la aplicación y de la persona usuaria final; asimismo, es importante que las aplicaciones de software requieran poco esfuerzo físico de la persona usuaria, no recargue la memoria de trabajo y sea fácil e intuitiva de usar. Por lo anterior, es preciso evaluar los diseños realizados con métodos de evaluación de usabilidad en donde participen usuarios y usuarias potenciales de la aplicación (Ferré, 2005). Por eso, en la etapa 3 el alumnado debe evaluar el diseño de la interfaz con la ayuda de una persona usuaria.

Etapa 3: Evaluación de la facilidad de uso del diseño de la interfaz. Después de implementar el diseño de la Interfaz el estudiantado debe evaluarlo. Según ISO (1999), la evaluación puede usarse para:

1. Proporcionar retroalimentación que sirva para la mejora del diseño.

2. Evaluar si los objetivos de las personas usuarias meta y de las organizaciones se han alcanzado.

3. Monitorizar el uso a largo plazo del producto o sistema.

En esta etapa cada grupo debe buscar una persona usuaria final para realizar la evaluación de la facilidad de uso del diseño de la interfaz. La persona usuaria final seleccionada debe cumplir con las características del enunciado del caso de estudio. Para realizar la evaluación se utilizó la técnica del SUS.

El SUS es un cuestionario que consta de diez preguntas y presenta como resultado un valor numérico a la apreciación subjetiva de la facilidad de uso de un sistema. La escala SUS se aplica después de que la persona usuaria ha tenido la oportunidad de utilizar la aplicación y realizar alguna tarea específica, pero antes de realizar algún informe o discusión acerca de la aplicación (Brooke, 1996; W. Y. Ng, WC. Lo y HS. Chan, 2011).

Se decidió usar esta técnica debido a que las preguntas de la encuesta cubren una variedad de aspectos de la usabilidad de los sistemas, tales como la necesidad de apoyo, la capacitación y la complejidad de la aplicación (W. Y. Ng et al., 2011). Asimismo Bangor, Kortum y Miller indican que el SUS es una herramienta muy robusta y versátil para profesionales de la usabilidad y que registra rápida y fácilmente la calificación subjetiva de las personas usuarias respecto de la facilidad de uso de un producto (2008). Además, la técnica es independiente de la tecnología que se utilice en el diseño de la interfaz (Bangor, Kortum y Miller, 2009) y se adecúa a los tiempos asignados a esta actividad académica.

El grupo de estudiantes lee y explica cada una de las preguntas y solicita a la persona usuaria final su respuesta inmediatamente. Es importante contestar todas las preguntas de la 
encuesta; pero, cuando la persona usuaria no se siente en capacidad de responder alguna pregunta en particular, se debe señalar el valor central de la escala (Brooke, 1996; Lewis y Sauro, 2009).

El SUS produce un solo número como resultado de la evaluación, el cual representa una medida compuesta de la usabilidad general del sistema en estudio (Bangor et al., 2009; Brooke, 1996). Si el resultado presentado por el SUS está por debajo de 60, indica que la usabilidad general del sistema es relativamente pobre. Por otro lado, se puede considerar que la usabilidad general del sistema es muy buena, si el resultado es mayor a 80 (Tullis y Albert, 2008). Sin embargo, si el resultado está entre 60 y 80 no se puede dar una valoración acerca de la usabilidad general de la aplicación.

Después de la evaluación, cada grupo realiza un informe escrito con los resultados obtenidos en la evaluación del diseño de la interfaz. Además, el alumnado debe mencionar posibles soluciones de usabilidad, si los resultados de la evaluación no fueron adecuados. En la figura 3 se presentan los pasos seguidos en la tercera etapa del caso de estudio.

Etapa 4: Presentación de los resultados a la clase. En la última etapa del caso de estudio se presenta el informe escrito a la clase, específicamente se exponen los resultados y posibles soluciones a los problemas encontrados en la evaluación del diseño de la interfaz.

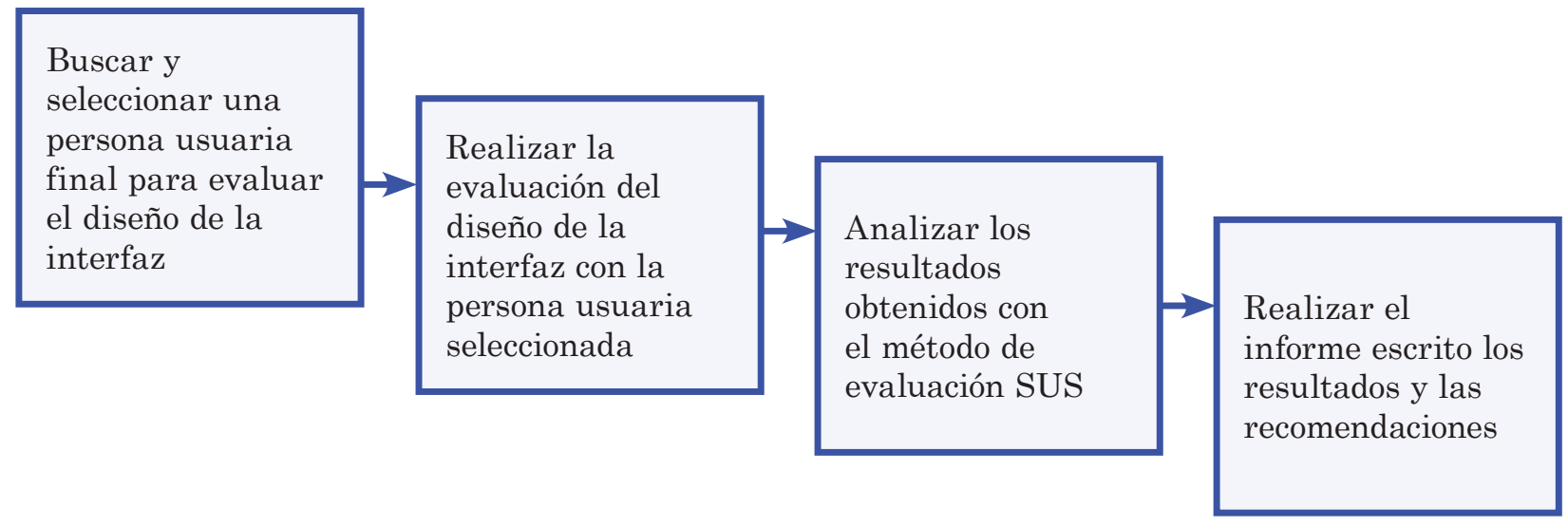

Figura 3. Pasos de la etapa 3: Evaluación de la facilidad de uso del diseño de la interfaz.

Elaboración propia (2012).

Para este punto cada grupo tiene 10 minutos para presentar el informe escrito y 10 minutos para una discusión sobre los resultados obtenidos y las soluciones propuestas. En esta parte el cuerpo docente media y guía la actividad.

En la presentación de los resultados se desea lograr que el alumnado pueda:

1. Interactuar con el resto de miembros de la clase.

2. Realizar aportes o intervenciones adecuadas durante la discusión. 
3. Medir la capacidad para distinguir entre diferentes tipos de datos (por ejemplo: hechos, opiniones, creencias, conceptos).

4. Explicar sus puntos de vista de manera adecuada y justificada.

\section{Tiempo utilizado para la aplicación del caso de estudio}

El tiempo utilizado para aplicar el caso de estudio se presenta en tabla 1, donde se muestra la distribución de los tiempos de cada actividad.

Tabla 1

Distribución del tiempo en la aplicación del caso de estudio

\begin{tabular}{ll}
\hline Actividad & Tiempo asignado a las actividades \\
\hline Entrega y explicación del caso de estudio & 1 hora \\
\hline Implementar el diseño de la interfaz & 1 semana \\
\hline Evaluación del diseño de la interfaz & 4 días \\
\hline Elaboración del informe escrito & 1 semana \\
\hline Presentación de los resultados & 2 días \\
\hline
\end{tabular}

Nota: Elaboración propia (2012).

\section{Resultados del diseño de la interfaz por parte de las personas usuarias finales}

Los resultados obtenidos de las distintas evaluaciones realizadas con la técnica del SUS por las personas usuarias finales, se presentan en la figura 4 .

Resultados de la evaluación con

la técnica de SUS

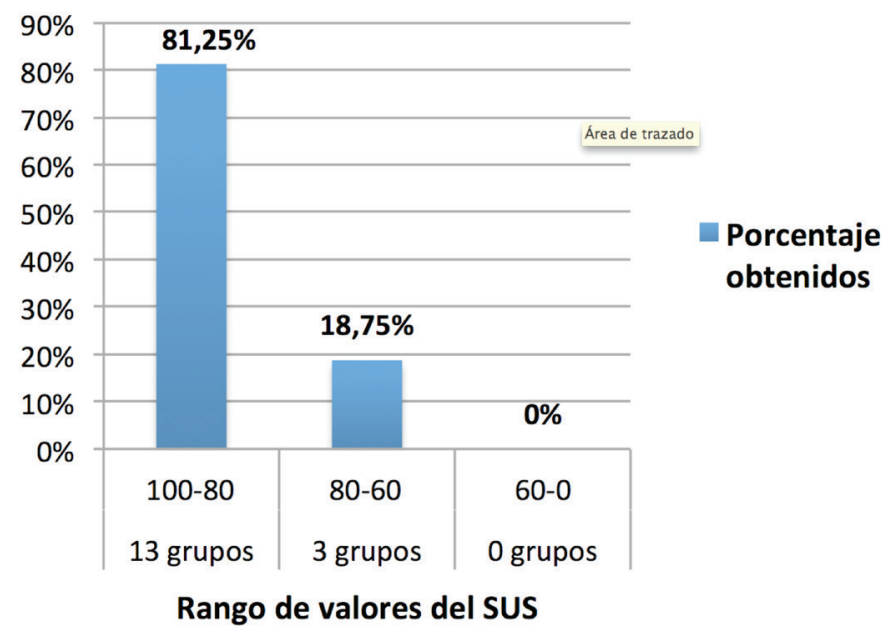

Figura 4. Resultados de la evaluación con la técnica del SUS. Elaboración propia (2012). 
En el gráfico de la figura 4 se observan los resultados de los 16 grupos que realizaron la evaluación del diseño de la interfaz de la aplicación web con una persona usuaria final, mediante la técnica del SUS. Los resultados obtenidos muestran que la usabilidad general de los sistemas diseñados por los grupos de estudiantes es muy buena, debido a que los resultados de 13 grupos (81.25\%) están en el rango de 80 a 100. En el rango de 0 a 60 no hubo ningún grupo con ese puntaje y de 60 a 80 estuvieron 3 grupos (18.75\%).

\section{Valoración de la estrategia didáctica}

En esta sección se presenta la valoración de aprendizaje al aplicar la estrategia didáctica. Para este punto se tomaron en cuenta los aprendizajes tanto del alumnado como del cuerpo docente.

\section{Valoración de la técnica en términos del aprendizaje del cuerpo docente}

El aprendizaje empezó desde el momento de seleccionar una estrategia didáctica acorde con lo que se quería lograr: incorporar usabilidad a un curso de aplicaciones web, ya que se debía analizar y optar por una estrategia didáctica que se acoplara al tema a desarrollar, al tipo de curso y al perfil del estudiantado.

El aprendizaje continuó con la planeación de cómo se iba a desarrollar la técnica, así como la cantidad de tiempo para la ejecución, la redacción del caso de estudio y la forma cómo se iba a evaluar la técnica por parte del alumnado. Realizar evaluaciones para cada una de las actividades, utilizar estas como herramientas para mejor la forma de impartir los temas y también la forma de redactar las descripciones de las actividades fueron los logros más significativos para el personal docente.

Durante la ejecución del caso de estudio se observó cómo el estudiantado mejoraba la experticia del tema a desarrollar y, en el diseño de la interfaz de la aplicación implementada, se vislumbraron mejoras significativas con respecto a la usabilidad.

\section{Valoración de la técnica en términos del aprendizaje del estudiantado}

Para evaluar la técnica en términos del aprendizaje se utilizó el instrumento de evaluación llamado curva de aprendizaje. Una curva de aprendizaje muestra la relación entre el tiempo de aprehensión, desarrollo y construcción de un conocimiento, contenido o competencia, y un número que representa el logro alcanzado en ese aspecto (Gutiérrez, Arias y Piedra, 2009). La curva de aprendizaje procura, en forma numérica, evaluar cómo los estudiantes autoperciben el desarrollo del aprendizaje de un contenido (Piedra, s. f.).

Esta técnica de evaluación se utilizó por varias razones: (1) permite al alumnado valorar su propio desempeño tanto en el trabajo individual como grupal (Gutiérrez et al., 2009), (2) provee un valor aproximado en algún momento de la actividad o indagación (Piedra, s. f.), (3) posibilita construir una curva de desarrollo a lo largo del tiempo para obtener el perfil del aprendizaje (Gutiérrez et al., 2009), en este caso del tema de usabilidad y (4) es una técnica que se aplica de manera sencilla y que proporciona una interpretación fácil de los resultados. 
Las preguntas de esta técnica se presentan a continuación:

A: Grado de conocimiento previo del tema de usabilidad, antes de que se impartiera la materia en clase.

B: Experticia que se tenía del contenido antes del desarrollo del caso de estudio y después de la explicación de la materia en clases.

C: Grado de asimilación e integración de los contenidos vistos en clase a los conocimientos previos en la elaboración del caso de estudio.

D: Experticia que se tiene ahora del contenido, después de aplicar el caso de estudio.

Todas las preguntas utilizan la escala que va del 1 al 10, donde el 10 la puntuación más alta y el 1 la más baja.

Para obtener la línea de la curva de aprendizaje del curso de Multimedios se aplicó la encuesta con las cuatro preguntas a cada estudiante, después de realizar el caso de estudio. La forma de aplicación de la encuesta se realizó de la siguiente manera:

1. En la clase siguiente a la terminación del caso de estudio, el personal docente explicó la técnica de la curva de aprendizaje y la forma de contestar las preguntas, además abarcó cualquier duda.

2. Se le entregó a cada estudiante la encuesta de la técnica de la curva de aprendizaje.

3. Cada estudiante contestó la encuesta.

4. Después de finalizar la encuesta se calculó la curva de aprendizaje.

En la figura 5 se puede observar los resultados obtenidos de la encuesta que se les aplicó a estudiantes del curso de Multimedios.

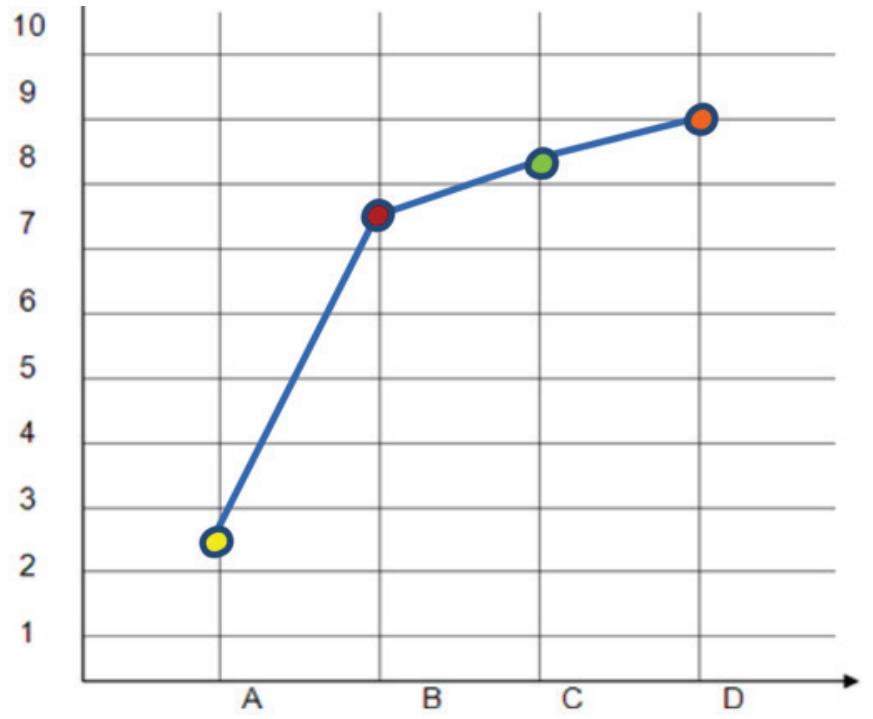

Figura 5. Resultados de la curva de aprendizaje.

Elaboración propia (2012). 
Como se puede apreciar en la figura 5, la mayoría de estudiantes no tenía conocimientos previos sobre el tema de usabilidad y evaluación de usabilidad en aplicaciones web, ya que el resultado de la pregunta $\mathrm{A}$ es de 2.4.

Cuando el tema se impartió en el aula, por medio de clases magistrales, el estudiantado aprendió definiciones, características y ejemplos del tema de usabilidad y de la evaluación del diseño de interfaces. En este punto se asignó una nota de 7.53, es decir, con las lecciones se logró un mejor entendimiento teórico del tema.

Para la pregunta $\mathrm{C}$ sobre el grado de asimilación del conocimiento previo y el visto en clase en la elaboración del caso de estudio, el alumnado asignó una nota de 8.27. Lo anterior quiere decir que se logró asimilar el tema impartido y se aplicó en la elaboración del diseño de la interfaz tomando en cuenta la tarea a realizar y la caracterización de la persona usuaria final.

$\mathrm{Y}$, por último, en la pregunta $\mathrm{D}$ sobre la experticia en el tema de usabilidad después de haber realizado el caso de estudio, los resultados fueron de 9.07. Esto indica que el estudiantado logró entender y aplicar adecuadamente el tema de usabilidad, por lo tanto, se alcanza el objetivo de la intención educativa.

\section{Conclusiones}

Con la aplicación del caso de estudio del tema de usabilidad en el curso de Multimedios se obtuvieron mejores resultados con respecto al diseño de la interfaz presentada por el alumnado. Se evidenció que las personas usuarias finales se sintieron a gusto con la aplicación. Lo anterior se demostró en las notas obtenidas en la evaluación con la técnica del SUS, donde el $81.25 \%$ de las interfaces se evaluó con nota superior o igual de $80 \%$, lo cual indica que la usabilidad general del sistema es muy buena.

Además, se pudo apreciar que el estudiantado entendía mejor las necesidades y características de las personas usuarias e incorporó mecanismos y estándares de usabilidad específicos para ellas. Las discusiones acerca de los resultados obtenidos en la evaluación del diseño de la interfaz en clases generaron conocimiento y desarrollaron la capacidad de retroalimentación del grupo.

El aplicar una estrategia didáctica mejora la curva de aprendizaje del tema a desarrollar. Asimismo, se realizan, de forma adecuada y ordenada, las distintas actividades que completan la estrategia didáctica, ya que se sigue un objetivo o meta, lo cual permite que el cuerpo docente y el alumnado no pierdan el rumbo de lo que se quiere lograr con la técnica utilizada.

Por último, el evaluar las distintas actividades del curso, en nuestro caso aplicar la encuesta sobre la experticia del tema de usabilidad, llega ser un aporte en la labor docente, ya que permite tomar en cuenta la opinión del alumnado, con el fin de tener retroalimentación para futuras actividades. 


\section{Referencias}

Bangor, A., Kortum, P. y Miller, J. (2008). An Empirical Evaluation of the System Usability Scale [Una evaluación empírica del sistema de escala de usabilidad]. International Journal of Human-computer Interaction - IJHCI, 24(6), 574-594.

Bangor, A., Kortum, P., y Miller, J. (2009). Determining What Individual SUS Scores Mean: Adding an Adjective Rating Scale [Determinando puntaciones promedias del SUS individuales: Agregando una escala de clasificación adjetivo]. Journal of Usability Studies, 4(3), 114-123. Recuperado de http://uxpajournal.org/wp-content/uploads/pdf/JUS Bangor May2009.pdf

Bolaños, J. D. (2013). Creación, historia y alcances de la carrera laboratorista químico en Costa Rica. Pensamiento Actual, 13(20), 25-41. Recuperado de http://revistas.ucr.ac.cr/ index.php/pensamiento-actual/article/view/15021

Brooke, J. (1996). SUS: a "quick and dirty" usability scale [SUS: un sistema de escala de usabilidad "rápido y sucio"]. En P. W. Jordan, B. Thomas, B. A. Weerdmeester, \& A. L. McClelland (Eds.), Usability Evaluation in Industry [Evaluación de usabilidad en la Industria]. London: Taylor and Francis. Recuperado de http://cui.unige.ch/isi/iclewiki/ media/ipm:test-suschapt.pdf

Castro, S. (2009). Costa Rica frente a la regionalización de la educación superior. El primer centro regional en San Ramón, Alajuela. InterSedes, 10(18), 174-204. Recuperado de http://www.intersedes.ucr.ac.cr/ojs/index.php/intersedes/article/viewFile/230/229

Dirección de Investigación y Desarrollo Educativo del Sistema. (2000). Las técnicas didácticas en el modelo educativo del TEC de Monterrey. Monterrey: México. Recuperado de http:/l sitios.itesm. $\mathrm{mx} / \mathrm{va} / \mathrm{dide} / \mathrm{docs}$ internos/inf-doc/tecnicas-modelo.PDF

Ferré, X. (2005). Marco de integración de la usabilidad en el proceso de desarrollo de software. (Tesis doctoral inédita). Universidad Politécnica de Madrid. Recuperado de http://oa.upm.es/440/1/XAVIER FERRE GRAU.PDF

Gutiérrez, M., Arias, J. y Piedra, L. (2009). Estrategias participativas para la enseñanza de las ciencias naturales en la Universidad de Costa Rica. Actualidades investigativas en educación, 9(2), 1-22. Recuperado de http://revista.inie.ucr.ac.cr/uploads/tx magazine/cie.pdf

International Organization for Standardization (ISO). (1998). ISO 9241-11 Ergonomic requirements for office work with visual display terminals (VDTs) - Part 11: Guidance on usability (1 $1^{\text {era }} \mathrm{ed}$.) [ISO 9241-11 requisitos ergonómico para trabajos de oficina con terminales de visualización de datos (PVD) - Parte 11: Orientación sobre la usabilidad (1 ${ }^{\text {era }}$ ed)]. Suiza. Recuperado de http://www.it.uu.se/edu/course/homepage/acsd/vt09/ISO9241part11.pdf

ISO. (1999). ISO 13407 Human centred design process for interactive systems [ISO 13407 Proceso de diseño centrado en el humano para sistemas interactivos]. Suiza. Recuperado de http:/l sophia.javeriana.edu.co/ cbustaca/HCI 2014 01/documentos/AENOR ISO-13407-2000.pdf 
Lewis, J. R. y Sauro, J. (2009). The factor structure of the system usability scale [El factor estructural del sistema de escala de usabilidad]. En Proceedings of the 1st International Conference on Human Centered Design: Held as Part of HCI International 2009. Denver, CO. doi: 10.1007/978-3-642-02806-9_12

Lorés, J, Granollers, T. y Lana, S. (2001). Introducción a la interacción persona-ordenador. En J. Lorés (Ed.), La interacción persona-ordenador (pp. 1-45). Lleida, España: AIPO, Asociación Interacción Persona Ordenador.

Lorés, J. y Granollers, T. (2004). La ingeniería de la usabilidad y de la accesibilidad aplicada al diseño y desarrollo de sitios web. Recuperado de https://utncomunicacionprofesional. files.wordpress.com/2012/04/ingenieria-de-sitios-web.pdf

Martínez, A. (1999). El estudio de casos como técnica didáctica -estudio de una experiencia en la enseñanza universitaria-. Innovación educativa, (9), 25-53. Recuperado de https:// dspace.usc.es/bitstream/10347/5188/1/pg 027-056 inneduc9.pdf

Piedra, L. (s. f.). Curva de aprendizaje. San José, Costa Rica. Recuperado del sitio Web del Departamento de Docencia Universitaria de la Universidad de Costa Rica. Recuperado de http://docenciauniversitaria.ucr.ac.cr/images/pdfs/texto/Practica curva de aprendizaje.pdf

Ramírez, W. (2012). La experiencia de usuario y la usabilidad. SCHEMA, (1), 67-86. Recuperado de http://www.polisemiadigital.com/schema/images/revista1/articulo04.pdf

Servicio de innovación educativa. (2008). Método del caso-Guías rápidas sobre innovación-. Recuperado de http://innovacioneducativa.upm.es/guias/MdC-guia.pdf

Tobón, S. (2005). Capítulo siete: Docencia Estratégica. En Formación Basada en Competencias (2da. ed., pp. 195-231). Bogotá: Ecoe Ediciones.

Universidad de Costa Rica, Sede de Occidente. (1998). Programa del curso: IF-7102Multimedios de la carrera Informática Empresarial. Grecia: Costa Rica.

Tullis, T., y Albert, B. (2008). Measuring the User Experience: Collecting, Analyzing, and Presenting Usability Metric [Medición de la Experiencia de Usuario: recopilación, análisis y presentación de Usabilidad Métricas]. Estados Unidos: Morgan Kaufmann Publishers.

W. Y. Ng, A., WC. Lo, H. y HS. Chan., A. (2011). Measuring the Usability of Safety Signs: A Use of System Usability Scale (SUS) [La medición de la usabilidad en señales de seguridad: Un uso del sistema de escala de usabilidad (SUS)]. En International Multi Congress of Engineers and Computer Scientists: Hong Kong, China. 